\title{
Caractérisation spatio-temporelle de la qualité de l'eau de la rivière Kinyankonge, affluent du Lac Tanganyika, Burundi
}

\author{
S. BUHUNGU ${ }^{1,2,3^{*}}$, E. MONTCHOWUI ${ }^{3}$, E. BARANKANIRA ${ }^{4}$, C. SIBOMANA ${ }^{2}$, \\ G. NTAKIMAZI ${ }^{2}$ et C.A. BONOU ${ }^{5}$
}

\author{
${ }^{I}$ Centre de Recherche en Sciences Naturelles et de l'Environnement, \\ Université du Burundi, BP 2700 Bujumbura, Burundi. \\ ${ }^{2}$ Département de Biologie, Faculté des Sciences, Université du Burundi. \\ ${ }^{3}$ Laboratoire de Recherche en Aquaculture et en Biologie et Ecologie Aquatiques, \\ Ecole d'Aquaculture de la Vallée, Université Nationale d'Agriculture, République du Bénin. \\ ${ }^{4}$ Département des Sciences Naturelles, École Normale Supérieure, \\ Boulevard du 28 Novembre, BP 6983, Bujumbura, Burundi. \\ ${ }^{5}$ Laboratoire de Recherche en Biologie Appliquée, Ecole Polytechnique d'Abomey-Calavi, \\ Université d'Abomey-Calavi, BP 2009 Cotonou, Bénin. \\ *Auteur correspondant ; E-mail : buhusimon@gmail.com
}

\section{REMERCIEMENTS}

Les auteurs remercient le Gouvernement du Burundi par son programme d'octroi des Bourses d'Etudes et des Stages pour avoir financé cette recherche.

\section{RESUME}

L'étude évalue l'état physico-chimique de la rivière Kinyankonge dans l'espace et dans le temps et l'impact des activités anthropiques. Des prélèvements mensuels d'eau ont été effectués dans sept stations d'échantillonnage pendant 18 mois. Les paramètres physico-chimiques ont été déterminés selon les méthodes de l'AFNOR. La charge organique dans la rivière a été évaluée à travers le calcul de l'Indice de Pollution Organique (IPO) de Leclercq. Les résultats ont révélé une variation spatio-temporelle de la pollution dans la rivière Kinyankonge avec de fortes proportions en saison pluvieuse. Cette pollution est d'origine domestique et provient également des effluents provenant de la Station d'épuration (STEP). Les résultats de l'analyse en composantes principales (ACP) sur les paramètres physico-chimiques ont montré deux groupes de stations. Le premier est constitué de quatre stations en amont caractérisées par la transparence et l'oxygène dissous les plus élevés. Le second est constitué de trois stations en aval avec des teneurs plus élevées en $\mathrm{DCO}, \mathrm{DBO}_{5}$, turbidité, alcalinité totale, $\mathrm{P}$ total, phosphates et en $\mathrm{pH}$. Comme l'ACP, l'indice de pollution organique compris entre 1,91 et 2,38 a catégorisé les stations en deux groupes, celles en amont présentant une forte pollution organique et celles en aval avec une très forte pollution organique.

(C) 2018 International Formulae Group. All rights reserved.

Mots clés : Pollution, physico-chimie, IPO, Rivière Kinyankonge, Burundi.

\section{Spatio-temporal characterization of the water quality of Kinyankonge River, a tributary of Lake Tanganyika, Burundi}




\begin{abstract}
This study assesses the physico-chemical state of the Kinyankonge River in space and in time and the impact of anthropogenic activities. Monthly water sampling was conducted at seven stations during 18 months. The physico-chemical parameters were analyzed according to AFNOR methods. The organic load in the river was assessed through the calculation of Leclercq's Organic Pollution Index (OPI). The results revealed a spatiotemporal variation in the pollution of the Kinyankonge River with high proportions in the rainy season. This pollution is from domestic origin and effluents from the Wastewater Treatment Plant (WWTP). The results of Principal component analysis (PCA) on physico-chemical parameters showed two groups of stations. The first consists of the four upstream stations characterized by highest transparency and dissolved oxygen. The second consists of the three downstream stations characterized by the highest values of COD, $\mathrm{BOD}_{5}$, turbidity, total alkalinity, total $\mathrm{P}$, phosphates and $\mathrm{pH}$. Like PCA results, the organic pollution index ranging from 1.91 to 2.38 , revealed two groups of the stations, the upstream stations with a strong organic pollution and the downstream stations with a very strong organic pollution.
\end{abstract}

(C) 2018 International Formulae Group. All rights reserved.

Keywords: Pollution, physical chemistry, OPI, Kinyankonge River, Burundi.

\section{INTRODUCTION}

Les écosystèmes d'eau douce sont des compartiments naturels nécessaires pour la continuité de la vie (Simpi et al., 2011). Ils sont essentiels pour diverses activités telles que l'approvisionnement des municipalités en eau potable, l'industrie, l'agriculture et les loisirs. Malheureusement, ils sont parmi les écosystèmes les plus sérieusement menacés par l'impact des activités anthropiques au cours de ce dernier siècle (Sala et al., 2000; Cox et al., 2002 ; Dudgeon et al., 2006). En effet, la croissance démographique accompagnée d'une urbanisation rapide cause de nombreuses perturbations pour les milieux naturels (Mc Kinney, 2002).

La rivière Kinyankonge traverse les quartiers Nord-Ouest de la ville de Bujumbura au Burundi. Elle se jette dans le Lac Tanganyika (le plus ancien des lacs africains avec une biodiversité remarquable) et constitue une source d'eau potable et de protéines animales pour une population d'environ 11 millions d'habitants. Les rejets domestiques, le lessivage des terres, les rejets d'une savonnerie et ceux d'une Station d'épuration de Buterere (STEP) constituent des sources majeures de pollution pour cette rivière. Ils constituent également une menace pour la qualité de l'eau et la biodiversité de cette rivière et du lac Tanganyika dont il est l'affluent.

Dès 1994, une station d'épuration est érigée au bord du lit principal de la rivière Kinyankonge et un canal y déverse ses eaux. Le projet avait été réalisé dans le but de collecter et de traiter toutes les eaux usées générées dans le périmètre urbain (Niyongabo, 2006), y compris les zones riveraines de la rivière. Bien que ce projet d'épuration fût salutaire pour la ville de Bujumbura; les ménages et industries, qui étaient ciblés n'ont malheureusement pas été tous connectés au réseau d'égout. En conséquence, la station d'épuration (STEP) est actuellement sous-exploitée. En principe, les STEP sont conçues pour épurer les eaux usées et limiter ainsi l'apport en excès de matière organique et de polluants dans le milieu naturel (Vindimian, 2006). Mais, plusieurs études ont montré que les rejets des stations d'épuration ont différents niveaux de charge polluante selon l'efficacité de la STEP, de façon que dans certains pays, les eaux usées traitées sont réutilisées pour l'irrigation des champs (Mohammad et Mazareh, 2003 ; AQUEREC Project, 2006).

Des études avaient montré que la station d'épuration de Buterere déverse ses effluents dans la rivière Kinyankonge qui devient un canal de transfert de polluants vers le lac Tanganyika qui est le milieu récepteur final (Niyungeko, 2011 ; Nsavyimana, 2014). De même, une étude préliminaire établissant le pollutogramme et l'hydrogramme de la rivière Kinyankonge a montré que les eaux sont fortement polluées (Buhungu et al., 2017). Un diagnostic plus complet incluant toutes les saisons hydrologiques de la situation actuelle de la pollution de la rivière s'avère nécessaire en vue d'un suivi de son évolution et des mesures visant l'amélioration 
de la qualité de l'eau et la protection de cet écosystème. La présente étude se propose d'évaluer, dans le temps et dans l'espace, l'impact des activités anthropiques sur la rivière Kinyankonge en se basant sur son état physico-chimique.

\section{MATERIEL ET METHODES}

\section{Zone d'étude et stations d'échantillonnage}

La rivière Kinyankonge a une longueur d'environ $6,5 \mathrm{~km}$. Elle traverse une localité très peu habitée et caractérisée par des étendues de terrains cultivables. Le sol est marécageux et donc favorable essentiellement à la culture du riz et du fourrage. Pour mener l'étude, sept stations d'échantillonnage ont été choisies en fonction de la diversité des rejets et des activités faites le long de son cours d'eau (Figure 1). La première station S1 ( $\mathrm{S}$ $3^{\circ} 20^{\prime} 22,765^{\prime \prime}$, E $29^{\circ} 21^{\prime} 10,655^{\prime \prime}$ et une altitude de $774.5 \mathrm{~m}$ ) est localisée en amont de la rivière Kinyankonge. Elle a été choisie dans le quartier Cibitoke pour rendre compte de l'état de la rivière à la source, les riverains y déversent les eaux usées et ordures ménagères. La seconde station $\mathrm{S} 2 \quad(\mathrm{~S}$ $3^{\circ} 20^{\prime} 30,527^{\prime \prime}$, E $29^{\circ} 21^{\prime} 27,655^{\prime \prime}$ et une altitude de $774.7 \mathrm{~m}$ ), quant à elle, est placée sur le canal Gikoma, pour rendre compte de la contribution de ce canal dans la charge polluante de la Kinyankonge. La troisième station S3 (S 320'43,598", E 29²1'27,468" et une altitude de $774.8 \mathrm{~m}$ ) est positionnée sur la rivière Nyabagere. Au bord de celle-ci, un nouveau quartier est en cours de construction ; le sable nécessaire est extrait dans la rivière Nyabagere. Les exploitants du sable y exercent une dégradation considérable du substrat important pour les organismes de la rivière Kinyankonge. Pour ce site, les échantillons permettent également d'évaluer la charge polluante déversée dans la rivière Kinyankonge. La quatrième station $\mathrm{S} 4$ (S $3^{\circ} 20^{\prime} 42,623^{\prime \prime}$, E $29^{\circ} 21^{\prime} 11,275^{\prime \prime}$ et une altitude de $771.3 \mathrm{~m}$ ) se trouve en aval des embouchures de la rivière Nyabagere et du canal Gikoma sur la rivière Kinyankonge. La cinquième station S5 (S 3०21'15,908", E $29^{\circ} 20^{\prime} 33,745^{\prime \prime}$ et une altitude de $765.6 \mathrm{~m}$ ) se trouve sur le canal de déversement des eaux de la STEP avant l'entrée de ces eaux dans la rivière Kinyankonge. La sixième station $\mathrm{S} 6$ ( $\mathrm{S}$ $3^{\circ} 21^{\prime} 16,657^{\prime \prime}$, E $29^{\circ} 20^{\prime} 32,535^{\prime \prime}$ et une altitude de $764.5 \mathrm{~m}$ ) se trouve sur la rivière
Kinyankonge après le point de déversement des eaux de la STEP. Elle reçoit les eaux du mélange de la station d'épuration et la rivière Kinyankonge. Quant à la station S7 (S $3^{\circ} 21^{\prime} 37,346^{\prime \prime}$, E $29^{\circ} 20^{\prime} 22,794^{\prime \prime}$ et une altitude de $760.5 \mathrm{~m}$ ), elle se trouve avant l'embouchure de la rivière Kinyankonge au Lac Tanganyika. Celle-ci contient les eaux de la rivière Kinyankonge et des effluents en provenance de l'usine de fabrication des savons qui y sont déversés après prétraitement physique.

\section{Echantillonnage}

Les prélèvements de l'eau ont été mensuels sur une période de 18 mois (de juillet 2015 à juin 2016, puis de janvier 2017 à juin 2017). Sur chacune des sept stations, un échantillon de $500 \mathrm{ml}$ chacun a été prélevé à $15 \mathrm{~cm}$ de profondeur, puis conditionné dans des flacons en polyéthylène. Les flacons préalablement nettoyés étaient rincés avec l'eau de la station avant le remplissage. Ces flacons contenant de l'eau brute de la rivière ont été immédiatement conservés dans des glaçons pour être transportés, puis mis au réfrigérateur $\left(4{ }^{\circ} \mathrm{C}\right)$ pour l'analyse des paramètres physico-chimiques non mesurés in-situ. Les prélèvements des échantillons destinés à l'analyse physico-chimique commençaient chaque fois à 7 heures pour se terminer à 11 heures.

\section{Mesure des paramètres physico-chimiques Matériel et techniques de mesure in-situ}

La température, le $\mathrm{pH}$, l'oxygène dissous, la conductivité électrique, la transparence de l'eau et les TDS ont été mesurés in-situ. La température a été mesurée avec un thermomètre portable à colonne de mercure gradué; le $\mathrm{pH}$, la conductivité électrique et le TDS ont été mesurés avec une multisonde Consort C6010 utilisant plusieurs électrodes spécifiques. L'électrode spécifique pour chaque paramètre a été plongée dans l'eau et le paramètre a été relevé sur l'écran électronique de l'appareil après stabilisation des valeurs. L'oxygène dissous a été mesuré avec l'oxymètre VWR 3000L.

La transparence a été mesurée avec le disque de Secchi. La profondeur de disparition et de réapparition du disque a permis de déterminer la valeur exacte de la transparence. La turbidité a été mesurée par un turbidimètre 
portable (TURBIQUANT @1100IR). Ainsi, un volume de $10 \mathrm{ml}$ d'eau brute a été mis dans un tube, après agitation manuelle; le tube a été placé dans l'appareil et la valeur de la turbidité a été lue sur l'écran du turbidimètre.

\section{Paramètres et méthodes d'analyse au laboratoire. \\ Les matières en suspension (MES)}

La détermination des matières en suspension dans l'eau a été effectuée par filtration sur membrane en fibres de verres selon la Norme AFNOR, NFT90- 105 (Rodier, 2005).

\section{Demande chimique en oxygène (DCO)}

La demande chimique en oxygène a été déterminée selon la méthode normalisée AFNOR (T90-101). Elle s'est déroulée en deux étapes. Une étape de la minéralisation dans un bloc chauffant avec des réactifs prédosés $\left(\mathrm{H}_{2} \mathrm{SO}_{4}, \mathrm{Ag}_{2} \mathrm{SO}_{4}, \mathrm{~K}_{2} \mathrm{SO}_{4}\right.$ et $\left.\mathrm{K}_{2} \mathrm{Cr}_{2} \mathrm{O}_{7}\right)$. Deux millilitres d'échantillon ont été additionnés de réactifs et portés à $148{ }^{\circ} \mathrm{C}$ pendant 2 heures dans un minéralisateur. La seconde étape a été celle de la mesure dans un photomètre PalinTest modèle DR 7500 à une longueur d'onde de $490 \mathrm{~nm}$.

\section{Demande biochimique en oxygène $\left(\mathrm{DBO}_{5}\right)$}

La demande biochimique en oxygène $a$ été mesurée à l'aide d'un DBO-mètre. Un volume d'eau correspondant à la gamme d'estimation de la $\mathrm{DBO}_{5}$ par rapport à la DCO du même échantillon a été versé dans le flacon en verre brun contenant un barreau magnétique. Deux pastilles de soude caustique $(\mathrm{NaOH})$ ont été ensuite introduites dans le godet en caoutchouc. La soude caustique a pour rôle d'absorber le $\mathrm{CO}_{2}$ produit lors de l'oxydation des matières organiques. La quantité d'oxygène est enregistrée toutes les 24 heures par l'OxiTop ${ }^{\mathrm{R}}$ sur une période de 5 jours. $\mathrm{La} \mathrm{DBO}_{5}$ exprimée en $\mathrm{mg} \mathrm{O}_{2} / \mathrm{l}$ est obtenue par multiplication de la valeur enregistrée au jour 5 par un facteur déterminé selon la gamme de DCO.

Les ammoniums, les nitrates, les nitrites, l'azote total, les phosphates et le phosphore total

Les paramètres comme les ions ammoniums, les nitrates, les nitrites, l'azote total, les orthophosphates, et le phosphore total ont été dosés par photométrie en utilisant le Photomètre Palin Test DR 7500, avec des réactifs pré-dosés.

\section{L'alcalinité totale}

L'alcalinité totale a été déterminée par titrimétrie selon les méthodes APHA (1995) et AFNOR NFT 90-003. Avec cette méthode, un volume de $100 \mathrm{ml}$ d'échantillon d'eau a été titré par une solution de $\mathrm{H}_{2} \mathrm{SO}_{4} 0,2 \mathrm{~N}$ après avoir ajouté à cette solution 6 gouttes de phénolphtaléine et 6 gouttes de méthyle orange comme indicateur. Le point d'équivalence a été atteint lorsque la couleur orange est apparue (Welch, 1948).

\section{La dureté totale}

La dureté totale a été déterminée par méthode de titrage (APHA, 1995). Un volume de $50 \mathrm{ml}$ d'échantillon d'eau a été titré par EDTA $(0,01 \mathrm{~N})$ après ajout de 3-4 gouttes de noir d'érichrome utilisé comme indicateur et 2 $\mathrm{ml}$ de tampon d'ammonium $(\mathrm{pH}=10)$. Le point d'équivalence a été atteint lorsque la coloration bleue est apparue.

\section{Analyse statistique}

Le test ANOVA de Kruskal-Wallis a été utilisé pour comparer les moyennes des différents paramètres physico-chimiques entre les stations et entre les saisons à l'aide du logiciel STATISTICA 7.1. Dans le but d'établir une relation entre les paramètres physico-chimiques des différentes stations, et pour mieux évaluer l'effet des activités anthropiques sur la qualité des eaux de la rivière Kinyankonge, une Analyse en Composantes Principales (ACP) a été appliquée à l'ensemble des paramètres afin de regrouper les stations polluées et non polluées. Cette analyse a été faite avec le logiciel CANOCO version 5.

L'Indice de Pollution Organique (IPO) de Leclercq (2001) a été également utilisé pour évaluer la charge organique dans la rivière. L'IPO classe la qualité des eaux en cinq (05) classes (Tableau 1). Cet indice s'obtient au moyen des valeurs des ammoniums, la $\mathrm{DBO}_{5}$, nitrites et phosphates. Le principe du calcul est de répartir les valeurs des quatre éléments polluants en cinq classes et de déterminer, à partir des valeurs obtenues dans l'étude, le numéro de classe correspondant pour chaque paramètre en se servant des données moyennes du Tableau 1. L'indice final est la moyenne des classes de pollution pour l'ensemble des paramètres. 


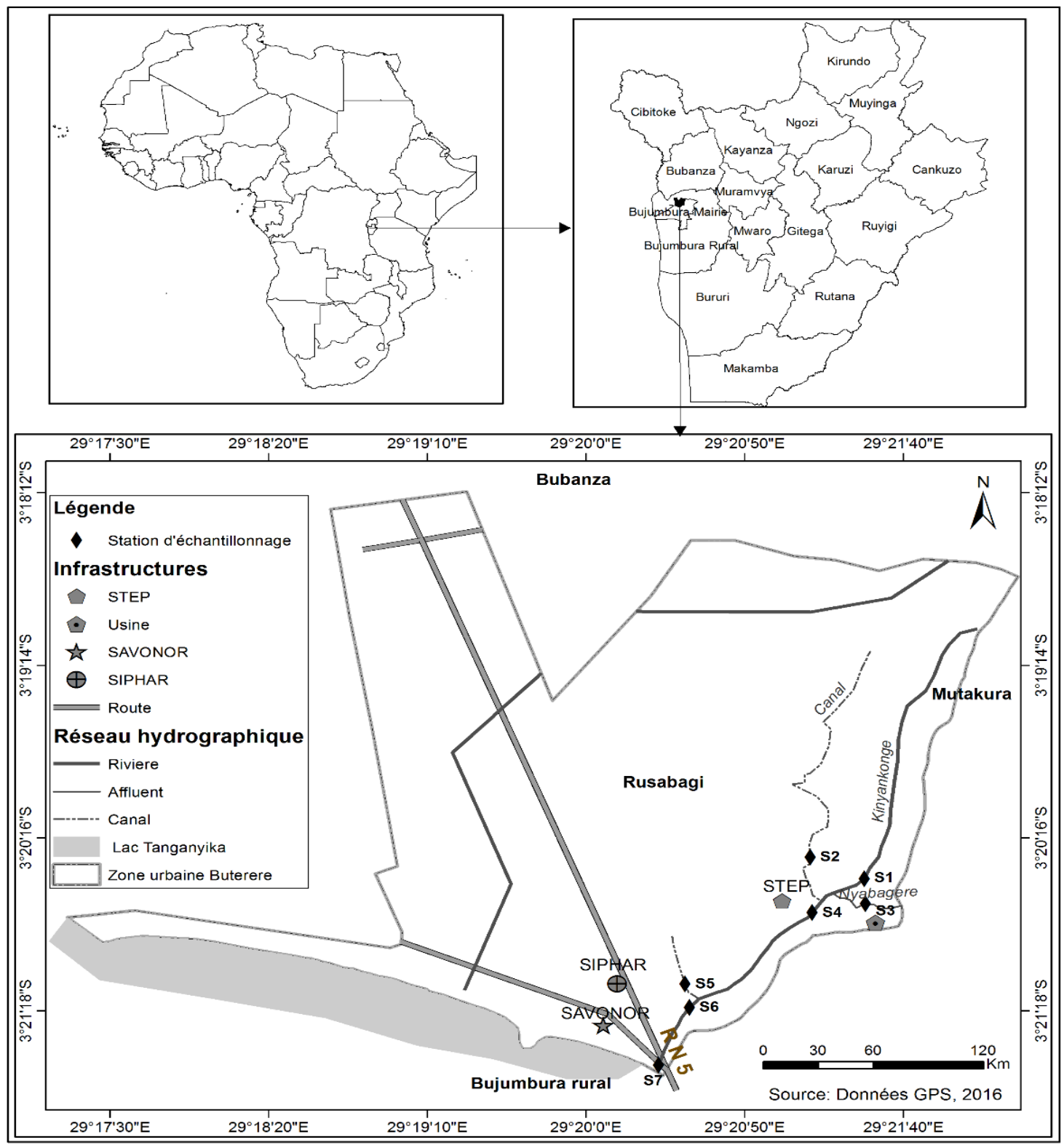

Figure 1 : Carte géographique des stations d'étude sur la rivière Kinyankonge.

Tableau 1 : Limites des classes de l'Indice de Pollution Organique (Leclercq, 2001).

\begin{tabular}{lccccc}
\hline Classes & $\mathbf{N H}_{\mathbf{4}}+(\mathbf{m g} / \mathbf{l})$ & $\mathbf{D B O}_{\mathbf{5}}\left(\mathbf{m g O}_{2} / \mathbf{l}\right)$ & $\mathbf{N O}_{2^{-}}(\boldsymbol{\mu g} / \mathbf{l})$ & $\mathbf{P O}_{\mathbf{4}}{ }^{3-}(\boldsymbol{\mu g} / \mathbf{l})$ & Classe de qualité IPO \\
\hline 5 & $<0,1$ & $<2$ & $<5$ & $<15$ & $4,6-5,0$ \\
4 & $0,1-0,9$ & $2,1-5$ & $6-10$ & $16-75$ & $4,0-4,5$ \\
3 & $1-2,4$ & $5,1-10$ & $11-50$ & $76-250$ & $3,0-3,9$ \\
2 & $2,5-6$ & $10,1-15$ & $51-150$ & $251-900$ & $2,0-2,9$ \\
1 & $>6$ & $>15$ & $>150$ & $>900$ & $1,0-1,9$ \\
\hline
\end{tabular}

(5) Pollution organique nulle, (4) pollution organique faible, (3) pollution organique modérée, (2) pollution organique forte, (1) pollution organique très forte. 


\section{RESULTATS}

\section{Variation spatiale des paramètres physico-chimiques}

Les valeurs moyennes des différents paramètres physico-chimiques mesurés dans les 7 stations sont présentées dans le Tableau 2. Aucune différence significative n'a été notée pour la température mesurée aux différentes stations $(\mathrm{p}>0,05)$. Pour le $\mathrm{pH}, \mathrm{la}$ valeur la plus élevée a été observée à la station S5, tandis que la plus basse a été observée à la station S2.

Les stations situées en amont ont été en général caractérisées par les teneurs en oxygène dissous les plus élevées. La cinquième station, quant à elle, a enregistré la moyenne la plus basse en oxygène dissous. $\mathrm{La}$ valeur de la conductivité électrique la plus élevée a été observée à la station S5 alors que la plus basse correspond à la station S3. La cinquième station a enregistré des teneurs en ions dissous les plus élevées.

Pour les solides totaux dissous (TDS), la moyenne la plus basse a été enregistrée à la station S2, la plus élevée à la station S5. Il a été remarqué que les solides totaux dissous et la conductivité électrique ont évolué dans le même sens. La moyenne de la transparence la plus basse a été notée à la station S5 et la plus élevée à la station $\mathrm{S} 1$. La moyenne de la turbidité la plus basse a été enregistrée à la station S1 et la plus élevée à la station S5. Pour ce paramètre, une évolution croissante a été observée de la première à la cinquième station. La turbidité et la transparence ont évolué en sens inverse.

Quant aux phosphates, la valeur moyenne minimale a été notée à la station S2 alors que la moyenne la plus élevée a été enregistrée à la station S5. Les moyennes minimale et maximale du phosphore total ont été enregistrées respectivement à la station S2 et à la station S5. Il a été remarqué que les phosphates et le phosphore total ont évolué dans le même sens. De même, la plus grande concentration en ammonium a été relevée à la station S7 et la plus petite aux stations S1 et S2. Une évolution croissante semble s'observer pour ce paramètre de la première à la septième station.
Pour les nitrites, aucune différence significative n'a été notée aux différentes stations ( $>>0,05)$. Une moyenne élevée pour les nitrates a été relevée à la station S5 et la plus basse à la station S4. Quant à l'azote total, une moyenne élevée a été notée à la station S5 et la plus basse à la station S3.

Les valeurs moyennes de l'alcalinité totale les plus basses ont été enregistrées à la station S3 et les plus élevées à la station S5. La valeur moyenne de la dureté totale la plus basse a été notée à la station S3 et la plus élevée à la station S5. Les MES ont été basses à la station S1 et élevées à la station S3. Enfin, pour la $\mathrm{DCO}$ et la $\mathrm{DBO}_{5}$, les valeurs moyennes ont été basses à la station $\mathrm{S} 1$ et élevées à la station S5. Ces deux paramètres ont évolué dans le même sens.

\section{Variation temporelle des paramètres physico-chimiques}

Le Tableau 3 présente la variation des paramètres physico-chimiques de la rivière Kinyankonge aux différents mois. Pour la température, la plus grande moyenne a été enregistrée au mois de juin 2017 et la plus petite en décembre 2015 correspondant à la période pluvieuse. Pour le $\mathrm{pH}$, la plus basse moyenne a été observée en décembre 2015 et la plus grande en août 2015. Il a été remarqué que le $\mathrm{pH}$ a augmenté de juillet-août (saison sèche) pour redescendre en octobrenovembre-décembre de la même année (saison de pluies). La moyenne de l'oxygène dissous était basse en mars 2016 et élevée en mai 2017. La conductivité électrique a connu une moyenne élevée en janvier 2017 et la plus basse en juin 2017. La plus grande moyenne de la transparence a été enregistrée en juin 2016 et la plus petite en octobre 2015, coïncidant alors au début des premières pluies. Il a été observé que les mois dont la transparence a été inférieure à $10 \mathrm{~cm}$ correspondaient aux mois de fortes pluies. La turbidité a connu la moyenne la plus basse en juin 2016 et la plus grande en octobre 2015. Il a été remarqué que la turbidité et la transparence ont évolué en sens inverse. Pour les mêmes mois, l'un a augmenté là où l'autre paramètre a diminué. 
Ils ont varié aussi bien dans l'espace que dans le temps. Quant aux nitrites, les valeurs minimale et maximale ont été enregistrées respectivement en juin 2016 et octobre 2015. Des teneurs élevées en azote total ont été observées en juin 2016 (saison sèche) et les plus basses en mars 2016 (saison pluvieuse). Le phosphore total a enregistré une valeur moyenne élevée en août 2015 et la plus basse en avril 2016.

Les stations et les saisons hydrologiques ont influencé significativement la variation de ce paramètre. L'alcalinité totale a enregistré une petite concentration en mai 2016 et la plus grande en octobre 2015. Aussi bien pour l'alcalinité, la saison sèche a été caractérisée par les valeurs les plus élevées tandis que la saison pluvieuse présente les valeurs les plus basses pour ce paramètre. Quant aux MES, la moyenne la plus basse a été enregistrée en octobre 2015 et la plus élevée en mars 2016.

Pour les TDS, les phosphates, l'ammonium, les nitrates, la dureté totale, la DCO et la $\mathrm{DBO}_{5}$, aucune différence significative n'a été notée $(p>0,05)$. Les paramètres $\mathrm{DCO}$ et $\mathrm{DBO}_{5}$ ont évolué dans le même sens.

\section{Dissimilarités des stations}

Une analyse en composantes principales (ACP) a été appliquée à l'ensemble des données et a permis de regrouper les stations selon leur niveau de pollution. Les deux premiers axes ont permis d'expliquer respectivement $66,02 \%$ et $20,89 \%$ de la dispersion globale des paramètres physico-chimiques relatifs aux stations, soit une variation totale de $86,91 \%$. Le premier axe factoriel oppose le groupe de variables (transparence, oxygène dissous) dont les valeurs élevées sont observées à la station $\mathrm{S} 1$ au groupe de variables $\left(\mathrm{DBO}_{5}, \mathrm{DCO}\right.$, turbidité) dont les valeurs élevées ont été observées à la station S5. Le deuxième axe factoriel, quant à lui, oppose la variable MES dont les valeurs élevées sont observées à la station S3 et la variable dureté totale dont les valeurs élevées sont enregistrées à la station S5. Pour les deux axes, les ions Nitrites sont mal représentés à cause de leurs faibles concentrations. La station S5 est caractérisée par de faibles valeurs en l'oxygène dissous. De même, la station $\mathrm{S} 1$ est caractérisée par une faible quantité de MES. La transparence, l'oxygène dissous et les nitrites sont négativement corrélés à l'axe 1 . Le deuxième axe divise les stations en deux grands groupes: les stations amont et les stations situées en aval de la rivière Kinyankonge.

La carte factorielle des variables et des individus montre que ces deux groupes de stations sont différents. Le premier groupe est composé des stations S1, S2, S3 et S4, situées en amont de la rivière Kinyankonge. Elles sont caractérisées par la transparence et l'oxygène dissous élevés. Ces deux paramètres sont faibles à la station S5.

Le deuxième groupe est composé des stations situées en aval de la rivière Kinyankonge. Il s'agit des stations S5, S6 et S7 qui sont caractérisées par les paramètres physico-chimiques tels la $\mathrm{DCO}$, la $\mathrm{DBO}_{5}$, la turbidité, l'alcalinité totale, le phosphates total et le $\mathrm{pH}$, dont les teneurs sont élevées (Figure 2).

Globalement, les deux axes montrent clairement que les stations situées en amont de la rivière (S1, S2, S3 et S4) sont moins polluées que les stations situées en aval de la rivière qui sont polluées (S5, S6 et S7). Les stations $\mathrm{S} 6$ et $\mathrm{S} 7$ sont localisées sur la rivière Kinyankonge après le canal de déversement des eaux en provenance de la station d'épuration de Buterere.

\section{Variabilité spatio-temporelle de la charge organique}

Les valeurs calculées de l'IPO ont montré que les eaux de la rivière Kinyankonge appartiennent à deux classes de pollution. Il s'agit de la pollution organique forte et la pollution organique très forte (Tableau 4).

L'analyse du Tableau 5 a montré que l'IPO a varié aussi bien dans l'espace que dans le temps. Ainsi, l'IPO a aussi catégorisé les stations en deux groupes tels que l'a montré l'ACP. Le groupe 1 est formé de stations amont (S1, S2, S3 et S4) dont l'indice a varié entre 2,1- 2,38. Elles sont de pollution organique forte tandis que le groupe 2 est constitué de stations aval (S5, S6 et S7) dont l'indice a varié de 1,91 à 1,95. Elles sont de très forte pollution organique. 
Tableau 2 : Variation spatiale des paramètres physico-chimiques.

\begin{tabular}{|c|c|c|c|c|c|c|c|c|c|}
\hline Paramètres & Station 1 & Station 2 & Station 3 & Station 4 & Station 5 & Station 6 & Station 7 & p-value & sign.p $<(0,05)$ \\
\hline $\mathrm{T}^{\circ} \mathrm{C}$ & $24,47 \pm 0,90$ & $24,45 \pm 1,13$ & $24,47 \pm 1,07$ & $25,01 \pm 1,12$ & $24,54 \pm 0,6$ & $24,39 \pm 0,70$ & $25,03 \pm 0,81$ & 0,21 & NS \\
\hline pH & $7,65 \pm 0,25$ & $7,45 \pm 0,25$ & $7,7 \pm 0,16$ & $7,59 \pm 0,24$ & $9,42 \pm 0,27$ & $8,09 \pm 0,57$ & $8,09 \pm 0,48$ & 0,00 & $S$ \\
\hline Ox dis. $\left(\mathrm{mgO}_{2} / \mathrm{l}\right)$ & $2,43 \pm 0,99$ & $2,22 \pm 1,55$ & $2,43 \pm 1,13$ & $2,19 \pm 0,94$ & $0,81 \pm 0,35$ & $1,69 \pm 0,93$ & $1,58 \pm 0,79$ & 0,00 & $S$ \\
\hline C. E. $(\mu \mathrm{S} / \mathrm{cm})$ & $684,05 \pm 164,8$ & $366,56 \pm 97,06$ & $345,8 \pm 95,3$ & $479,85 \pm 123,2$ & $1078,8 \pm 225,1$ & $628 \pm 242,4$ & $615,27 \pm 176,7$ & 0,00 & S \\
\hline TDS (mg/l) & $430,05 \pm 83,8$ & $191,36 \pm 42,06$ & $194,31 \pm 62,9$ & $265,23 \pm 70,5$ & $600,7 \pm 92,4$ & $339,2 \pm 93,3$ & $331,96 \pm 75,6$ & 0,00 & S \\
\hline Trans.(cm) & $14,16 \pm 4,1$ & $12,57 \pm 3,7$ & $8,35 \pm 2,67$ & $11,01 \pm 3,74$ & $7,32 \pm 2,40$ & $10,7 \pm 3,56$ & $11,22 \pm 3,78$ & 0,00 & S \\
\hline Turb. (NTU) & $29,43 \pm 16,3$ & $61,34 \pm 22,5$ & $114,27 \pm 84,6$ & $113,17 \pm 75,1$ & $266,05 \pm 82,7$ & $113,59 \pm 82,8$ & $93,98 \pm 61,1$ & 0,00 & S \\
\hline $\mathrm{PO}_{4}^{-3}(\mathrm{mg} / \mathrm{l})$ & $1,36 \pm 0,33$ & $1,04 \pm 0,26$ & $1,25 \pm 0,38$ & $1,26 \pm 0,39$ & $6,29 \pm 2,15$ & $2,76 \pm 2,03$ & $3,19 \pm 1,83$ & 0,00 & $S$ \\
\hline $\mathrm{NH}_{4}^{+}(\mathrm{mg} / \mathrm{l})$ & $0,74 \pm 0,40$ & $0,76 \pm 0,40$ & $0,67 \pm 0,42$ & $1,06 \pm 0,31$ & $1,86 \pm 0,83$ & $1,64 \pm 0,93$ & $1,88 \pm 0,70$ & 0,00 & $S$ \\
\hline $\mathrm{NO}_{3}^{-}(\mathrm{mg} / \mathrm{l})$ & $1,16 \pm 0,36$ & $1,03 \pm 0,26$ & $0,96 \pm 0,34$ & $0,94 \pm 0,39$ & $1,83 \pm 0,91$ & $1,56 \pm 0,76$ & $1,44 \pm 0,68$ & 0,00 & S \\
\hline $\mathrm{NO}_{2}^{-}(\mathrm{mg} / \mathrm{l})$ & $0,03 \pm 0,02$ & $0,03 \pm 0,01$ & $0,03 \pm 0,02$ & $0,03 \pm 0,02$ & $0,017 \pm 0,01$ & $0,016 \pm 0,01$ & $0,037 \pm 0,03$ & 0,05 & NS \\
\hline Ntot(mg/l) & $2,56 \pm 0,88$ & $2,03 \pm 0,85$ & $1,97 \pm 0,61$ & $2,14 \pm 0,52$ & $2,96 \pm 1,02$ & $2,6 \pm 1,05$ & $2,65 \pm 0,90$ & 0,02 & $\mathrm{~S}$ \\
\hline Ptot (mg/l) & $1,56 \pm 0,64$ & $1,27 \pm 0,48$ & $1,71 \pm 0,95$ & $1,56 \pm 0,64$ & $3,33 \pm 1,06$ & $2,47 \pm 0,83$ & $2,31 \pm 0,93$ & 0,00 & $S$ \\
\hline Alc tot (mg/l) & $251,88 \pm 92,4$ & $150,31 \pm 52,0$ & $137,33 \pm 39,9$ & $167,2 \pm 81,1$ & $527,6 \pm 78,9$ & $254,66 \pm 53,6$ & $275,44 \pm 78,3$ & 0,00 & S \\
\hline Dur tot $(\mathrm{mg} / \mathrm{l})$ & $232,72 \pm 80,4$ & $134,43 \pm 54,5$ & $106,5 \pm 19,7$ & $145,5 \pm 63,5$ & $237,2 \pm 33,4$ & $183,33 \pm 54,1$ & $184,22 \pm 48,1$ & 0,00 & S \\
\hline MES (mg/l) & $29,58 \pm 13,2$ & $75,83 \pm 32,9$ & $229 \pm 67,8$ & $164,4 \pm 55,1$ & $192,61 \pm 47,0$ & $139,38 \pm 64,3$ & $144,5 \pm 58,1$ & 0,00 & S \\
\hline $\mathrm{DCO}\left(\mathrm{mgO}_{2} / \mathrm{l}\right)$ & $42,94 \pm 24,2$ & $75,93 \pm 33,9$ & $116,55 \pm 53,6$ & $120,05 \pm 97,2$ & $279,61 \pm 63,3$ & $139,5 \pm 68,8$ & $155,5 \pm 54,2$ & 0,00 & S \\
\hline $\mathrm{DBO}_{5}\left(\mathrm{mgO}_{2} / \mathrm{l}\right)$ & $21,94 \pm 11,1$ & $37,62 \pm 16,1$ & $58,88 \pm 26,3$ & $59,72 \pm 46,6$ & $139,16 \pm 31,6$ & $71,66 \pm 37,6$ & $78,61 \pm 29,1$ & 0,00 & $\mathrm{~S}$ \\
\hline
\end{tabular}


Tableau 3 : Variabilité temporelle des paramètres physico-chimiques.

\begin{tabular}{|c|c|c|c|c|c|c|c|}
\hline Paramètres & Juillet-15 & Août-15 & Septembre-15 & Octobre-15 & Novembre-15 & Décembre-15 & Janvier-16 \\
\hline $\mathrm{T}^{\circ} \mathrm{C}$ & $23,8 \pm 1,15$ & $24,3 \pm 0,63$ & $24,9 \pm 0,85$ & $24,5 \pm 0,88$ & $23,5 \pm 0,52$ & $22,5 \pm 0,61$ & $25,3 \pm 0,30$ \\
\hline $\mathrm{pH}$ & $8,19 \pm 0,54$ & $8,47 \pm 0,64$ & $8,25 \pm 0,57$ & $7,8 \pm 0,83$ & $7,61 \pm 0,69$ & $7,48 \pm 0,69$ & $8,03 \pm 0,79$ \\
\hline $\mathrm{Ox}$ dis. $\left(\mathrm{mgO}_{2} / 1\right)$ & $2,04 \pm 0,83$ & $1,52 \pm 1,01$ & $1,98 \pm 0,59$ & $2,69 \pm 1,09$ & $2,3 \pm 0,96$ & $2,32 \pm 1,07$ & $2,07 \pm 1,19$ \\
\hline C. E. $(\mu \mathrm{S} / \mathrm{cm})$ & $708,1 \pm 340,0$ & $795,6 \pm 304,1$ & $823,6 \pm 292,2$ & $568 \pm 360,0$ & $553,2 \pm 336,2$ & $566 \pm 271,9$ & $557,7 \pm 182,9$ \\
\hline TDS (mg/l) & $351,5 \pm 141,1$ & $481,1 \pm 156,0$ & $482,8 \pm 186,9$ & $305 \pm 182,9$ & $297,1 \pm 183,1$ & $298,1 \pm 154,7$ & $305,7 \pm 108,4$ \\
\hline Trans. (cm) & $13,57 \pm 2,57$ & $12,86 \pm 2,43$ & $10,41 \pm 3,58$ & $4,74 \pm 1,15$ & $5,07 \pm 1,13$ & $9,42 \pm 4,27$ & $11,85 \pm 2,89$ \\
\hline Turb. (NTU) & $63,55 \pm 53,05$ & $68,31 \pm 52,59$ & $106,1 \pm 111,4$ & $185,4 \pm 118,7$ & $166,5 \pm 87,5$ & $116,6 \pm 99,3$ & $77,2 \pm 57,77$ \\
\hline $\mathrm{PO}_{4}^{-3}(\mathrm{mg} / \mathrm{l})$ & $2,23 \pm 1,31$ & $1,83 \pm 0,61$ & $1,7 \pm 0,35$ & $1,55 \pm 0,23$ & $2,38 \pm 2,50$ & $2,89 \pm 2,99$ & $1,87 \pm 1,42$ \\
\hline $\mathrm{NH}_{4}^{+}(\mathrm{mg} / \mathrm{l})$ & $1,16 \pm 0,95$ & $1,28 \pm 1,0$ & $1,26 \pm 0,92$ & $1,21 \pm 0,96$ & $1,96 \pm 0,93$ & $1,37 \pm 1,46$ & $0,76 \pm 0,34$ \\
\hline $\mathrm{NO}_{3}{ }^{-}(\mathrm{mg} / \mathrm{l})$ & $1,34 \pm 0,35$ & $1,67 \pm 0,36$ & $1,22 \pm 0,52$ & $1,53 \pm 0,68$ & $1,25 \pm 0,72$ & $1,15 \pm 0,32$ & $1,15 \pm 0,51$ \\
\hline $\mathrm{NO}_{2}^{-}(\mathrm{mg} / \mathrm{l})$ & $0,041 \pm 0,01$ & $0,034 \pm 0,01$ & $0,032 \pm 0,01$ & $0,064 \pm 0,02$ & $0,028 \pm 0,01$ & $0,039 \pm 0,03$ & $0,057 \pm 0,02$ \\
\hline Ntot (mg/l) & $2,10 \pm 0,72$ & $2,56 \pm 0,81$ & $2,28 \pm 0,80$ & $2,62 \pm 0,56$ & $2,97 \pm 0,82$ & $2,10 \pm 0,83$ & $1,72 \pm 0,88$ \\
\hline Ptot (mg/l) & $2,20 \pm 0,68$ & $3,50 \pm 0,42$ & $2,83 \pm 1,0$ & $1,59 \pm 0,46$ & $2,76 \pm 1,1$ & $2,54 \pm 1,61$ & $1,84 \pm 0,79$ \\
\hline Alc tot $(\mathrm{mg} / \mathrm{l})$ & $320,7 \pm 157$ & $370,5 \pm 157,1$ & $336 \pm 156,3$ & $373,5 \pm 141,0$ & $263,2 \pm 176,8$ & $228,5 \pm 117,3$ & $232,8 \pm 124,4$ \\
\hline Dur tot $(\mathrm{mg} / \mathrm{l})$ & $219,2 \pm 63,8$ & $238,8 \pm 70,4$ & $229 \pm 85,1$ & $282,4 \pm 63,4$ & $167,7 \pm 43,4$ & $137,2 \pm 41,6$ & $135 \pm 35,3$ \\
\hline MES (mg/l) & $100,8 \pm 84,8$ & $134,1 \pm 118,2$ & $124,8 \pm 84,6$ & $89,4 \pm 64,5$ & $133,5 \pm 85,3$ & $157,2 \pm 101,6$ & $120,1 \pm 47,8$ \\
\hline $\mathrm{DCO}\left(\mathrm{mgO}_{2} / \mathrm{l}\right)$ & $117,7 \pm 107,2$ & $145,3 \pm 128,4$ & $114,1 \pm 83,2$ & $137,7 \pm 80,3$ & $161,1 \pm 127,3$ & $148,5 \pm 90,7$ & $90,5 \pm 50,1$ \\
\hline $\mathrm{DBO}_{5}\left(\mathrm{mgO}_{2} / \mathrm{l}\right)$ & $59,2 \pm 52,2$ & $71,6 \pm 62,9$ & $57,5 \pm 39,1$ & $66,4 \pm 36,3$ & $77,8 \pm 57,2$ & $73,5 \pm 43,8$ & $44,2 \pm 24,3$ \\
\hline
\end{tabular}


Tableau 3 : Variabilité temporelle des paramètres physico-chimiques (suite).

\begin{tabular}{|c|c|c|c|c|c|c|c|}
\hline Param. & Février-16 & Mars-16 & Avril-16 & Mai-16 & Juin-16 & Janvier-17 & Février-17 \\
\hline $\mathrm{T}^{\circ} \mathrm{C}$ & $24,6 \pm 0,44$ & $24,9 \pm 0,34$ & $25,04 \pm 0,77$ & $24,5 \pm 0,46$ & $25,2 \pm 0,81$ & $25,08 \pm 0,47$ & $24,9 \pm 0,56$ \\
\hline $\mathrm{pH}$ & $7,99 \pm 0,51$ & $8,12 \pm 0,81$ & $7,93 \pm 0,67$ & $7,79 \pm 0,66$ & $7,86 \pm 0,56$ & $8,15 \pm 0,86$ & $8,13 \pm 0,71$ \\
\hline Ox dis. $\left(\mathrm{mgO}_{2} / \mathrm{l}\right)$ & $1,41 \pm 0,50$ & $0,95 \pm 0,39$ & $1 \pm 0,14$ & $1,1 \pm 0,46$ & $2,47 \pm 1,00$ & $2 \pm 1,15$ & $1,4 \pm 0,62$ \\
\hline C. E. $(\mu \mathrm{S} / \mathrm{cm})$ & $563,7 \pm 167,9$ & $573,8 \pm 262,2$ & $502,7 \pm 259,6$ & $479,8 \pm 305,7$ & $621,7 \pm 301,9$ & $895,8 \pm 417,5$ & $582,7 \pm 192,5$ \\
\hline TDS (mg/l) & $305,5 \pm 128,6$ & $309,3 \pm 149,4$ & $273,7 \pm 135,4$ & $253,8 \pm 166,5$ & $348,5 \pm 182,5$ & $315,2 \pm 101,8$ & $313,7 \pm 145,1$ \\
\hline Trans. (cm) & $12,91 \pm 3,80$ & $7,44 \pm 2,06$ & $13,78 \pm 3,71$ & $7,58 \pm 1,03$ & $15,14 \pm 2,69$ & $11,71 \pm 2,94$ & $11,95 \pm 3,29$ \\
\hline Turb. (NTU) & $67,51 \pm 49,72$ & $112,1 \pm 79,15$ & $74,01 \pm 79,89$ & $105,8 \pm 58,01$ & $54,89 \pm 53,55$ & $260,5 \pm 141,3$ & $69,77 \pm 51,00$ \\
\hline $\mathrm{PO}_{4}^{-3}(\mathrm{mg} / \mathrm{l})$ & $2,2 \pm 1,95$ & $2,68 \pm 2,83$ & $2,18 \pm 1,89$ & $2,44 \pm 2,1$ & $2,14 \pm 2,63$ & $2,15 \pm 1,60$ & $3,24 \pm 2,72$ \\
\hline $\mathrm{NH}_{4}^{+}(\mathrm{mg} / \mathrm{l})$ & $1,12 \pm 0,62$ & $1,21 \pm 1,13$ & $1,20 \pm 0,37$ & $1,43 \pm 0,75$ & $1,25 \pm 0,84$ & $0,83 \pm 0,44$ & $0,91 \pm 0,34$ \\
\hline $\mathrm{NO}_{3}^{-}(\mathrm{mg} / \mathrm{l})$ & $0,74 \pm 0,32$ & $1,12 \pm 0,21$ & $1,05 \pm 0,17$ & $1,13 \pm 0,31$ & $0,80 \pm 0,38$ & $1,3 \pm 0,62$ & $1,01 \pm 0,35$ \\
\hline $\mathrm{NO}_{2}^{-}(\mathrm{mg} / \mathrm{l})$ & $0,047 \pm 0,03$ & $0,013 \pm 0,01$ & $0,028 \pm 0,01$ & $0,031 \pm 0,02$ & $0,011 \pm 0,01$ & $0,039 \pm 0,03$ & $0,019 \pm 0,01$ \\
\hline Ntot $(\mathrm{mg} / \mathrm{l})$ & $2,29 \pm 0,90$ & $1,68 \pm 0,78$ & $2,8 \pm 0,74$ & $3,17 \pm 0,76$ & $3,18 \pm 0,66$ & $1,8 \pm 0,81$ & $2,48 \pm 1,12$ \\
\hline Ptot (mg/l) & $1,63 \pm 1,21$ & $1,89 \pm 0,93$ & $1,42 \pm 0,56$ & $1,85 \pm 0,58$ & $1,53 \pm 0,51$ & $1,9 \pm 0,98$ & $1,79 \pm 1,60$ \\
\hline Alc tot (mg/l) & $217,5 \pm 114,2$ & $203,4 \pm 122,4$ & $201,5 \pm 117,3$ & $200,4 \pm 129,8$ & $243,1 \pm 188,7$ & $215,8 \pm 120,9$ & $212,2 \pm 128,9$ \\
\hline Dur tot (mg/l) & $143,2 \pm 44,5$ & $159 \pm 76,2$ & $144,5 \pm 40,3$ & $144,5 \pm 34,3$ & $147,7 \pm 75,3$ & $156 \pm 60,7$ & $153,4 \pm 54,5$ \\
\hline MES (mg/l) & $152,5 \pm 74,6$ & $189,1 \pm 116,9$ & $141,5 \pm 67,9$ & $183,1 \pm 72,7$ & $96,2 \pm 43,8$ & $131 \pm 54,9$ & $161,1 \pm 80,1$ \\
\hline $\mathrm{DCO}\left(\mathrm{mgO}_{2} / \mathrm{l}\right)$ & $103,2 \pm 60,0$ & $100,1 \pm 77,2$ & $92,5 \pm 62,5$ & $112,8 \pm 57,3$ & $73,7 \pm 74,4$ & $215,7 \pm 89,6$ & $114,8 \pm 74,2$ \\
\hline $\mathrm{DBO}_{5}\left(\mathrm{mgO}_{2} / \mathrm{l}\right)$ & $49,2 \pm 29,2$ & $47,1 \pm 37,6$ & $46,4 \pm 29,9$ & $60,7 \pm 29,7$ & $37,1 \pm 38,4$ & $105 \pm 43,7$ & $58,5 \pm 41,1$ \\
\hline
\end{tabular}


Tableau 3 : Variabilité temporelle des paramètres physico-chimiques (suite et fin)

\begin{tabular}{|c|c|c|c|c|c|c|}
\hline Paramètres & Mars-17 & Avril-17 & Mai-17 & Juin-17 & p-value & sign.p $<(0,05)$ \\
\hline $\mathrm{T}^{\circ} \mathrm{C}$ & $24,7 \pm 0,42$ & $24,9 \pm 0,82$ & $24,6 \pm 0,50$ & $25,5 \pm 0,63$ & 0,0001 & $\mathrm{~S}$ \\
\hline $\mathrm{pH}$ & $8,21 \pm 0,94$ & $8,18 \pm 0,82$ & $8,01 \pm 0,73$ & $8,04 \pm 0,76$ & 0,01 & S \\
\hline Ox dis. $\left(\mathrm{mgO}_{2} / \mathrm{l}\right)$ & $0,97 \pm 0,35$ & $1,43 \pm 1,10$ & $3,61 \pm 1,52$ & $2,92 \pm 1,11$ & 0,00 & S \\
\hline C. E. $(\mu \mathrm{S} / \mathrm{cm})$ & $576,2 \pm 248,1$ & $595 \pm 289,2$ & $486,3 \pm 157,9$ & $471,9 \pm 201,4$ & 0,01 & S \\
\hline TDS (mg/l) & $367,4 \pm 155,6$ & $344,5 \pm 155,8$ & $437,2 \pm 124,4$ & $342,4 \pm 139,7$ & 0,29 & NS \\
\hline Trans. (cm) & $7,92 \pm 2,28$ & $11,58 \pm 3,86$ & $11,97 \pm 2,59$ & $13,54 \pm 3,18$ & 0,0001 & $\mathrm{~S}$ \\
\hline Turb. (NTU) & $120,4 \pm 71,55$ & $113,9 \pm 113,2$ & $144,2 \pm 113,0$ & $136,4 \pm 92,60$ & 0,001 & S \\
\hline $\mathrm{PO}_{4}^{-3}(\mathrm{mg} / \mathrm{l})$ & $3,49 \pm 3,53$ & $3,72 \pm 3,20$ & $2,96 \pm 2,64$ & $2,65 \pm 2,49$ & 0,59 & NS \\
\hline $\mathrm{NH}_{4}^{+}(\mathrm{mg} / \mathrm{l})$ & $1,11 \pm 0,55$ & $1,52 \pm 0,59$ & $1,42 \pm 0,51$ & $1,32 \pm 0,63$ & 0,09 & NS \\
\hline $\mathrm{NO}_{3}^{-}(\mathrm{mg} / \mathrm{l})$ & $1,75 \pm 1,01$ & $1,99 \pm 1,42$ & $1,42 \pm 0,62$ & $1,38 \pm 0,73$ & 0,06 & NS \\
\hline $\mathrm{NO}_{2}^{-}(\mathrm{mg} / \mathrm{l})$ & $0,012 \pm 0,006$ & $0,013 \pm 0,01$ & $0,013 \pm 0,01$ & $0,012 \pm 0,01$ & 0,0001 & S \\
\hline Ntot (mg/l) & $1,7 \pm 0,66$ & $2,8 \pm 1,15$ & $2,4 \pm 0,64$ & $2,99 \pm 0,78$ & 0,001 & S \\
\hline Ptot (mg/l) & $1,87 \pm 0,98$ & $2,22 \pm 1,34$ & $1,86 \pm 0,56$ & $1,86 \pm 0,85$ & 0,04 & S \\
\hline Alc tot $(\mathrm{mg} / \mathrm{l})$ & $265,2 \pm 164,8$ & $237,4 \pm 128,3$ & $232,5 \pm 138,1$ & $240,1 \pm 146,1$ & 0,02 & $\mathrm{~S}$ \\
\hline Dur tot $(\mathrm{mg} / \mathrm{l})$ & $210,4 \pm 94,7$ & $170,2 \pm 61,7$ & $178,1 \pm 56,0$ & $158,4 \pm 60,8$ & 0,207 & NS \\
\hline MES (mg/l) & $187,4 \pm 101,1$ & $131,8 \pm 68,7$ & $183,1 \pm 80,6$ & $105,7 \pm 53,6$ & 0,015 & $\mathrm{~S}$ \\
\hline $\mathrm{DCO}\left(\mathrm{mgO}_{2} / \mathrm{l}\right)$ & $144,8 \pm 105,5$ & $150,4 \pm 110,1$ & $234,2 \pm 103,7$ & $149,2 \pm 68,9$ & 0,77 & NS \\
\hline $\mathrm{DBO}_{5}\left(\mathrm{mgO}_{2} / \mathrm{l}\right)$ & $87,8 \pm 67,0$ & $78,8 \pm 56,3$ & $112,1 \pm 50,0$ & $76,4 \pm 34,9$ & 0,86 & NS \\
\hline
\end{tabular}




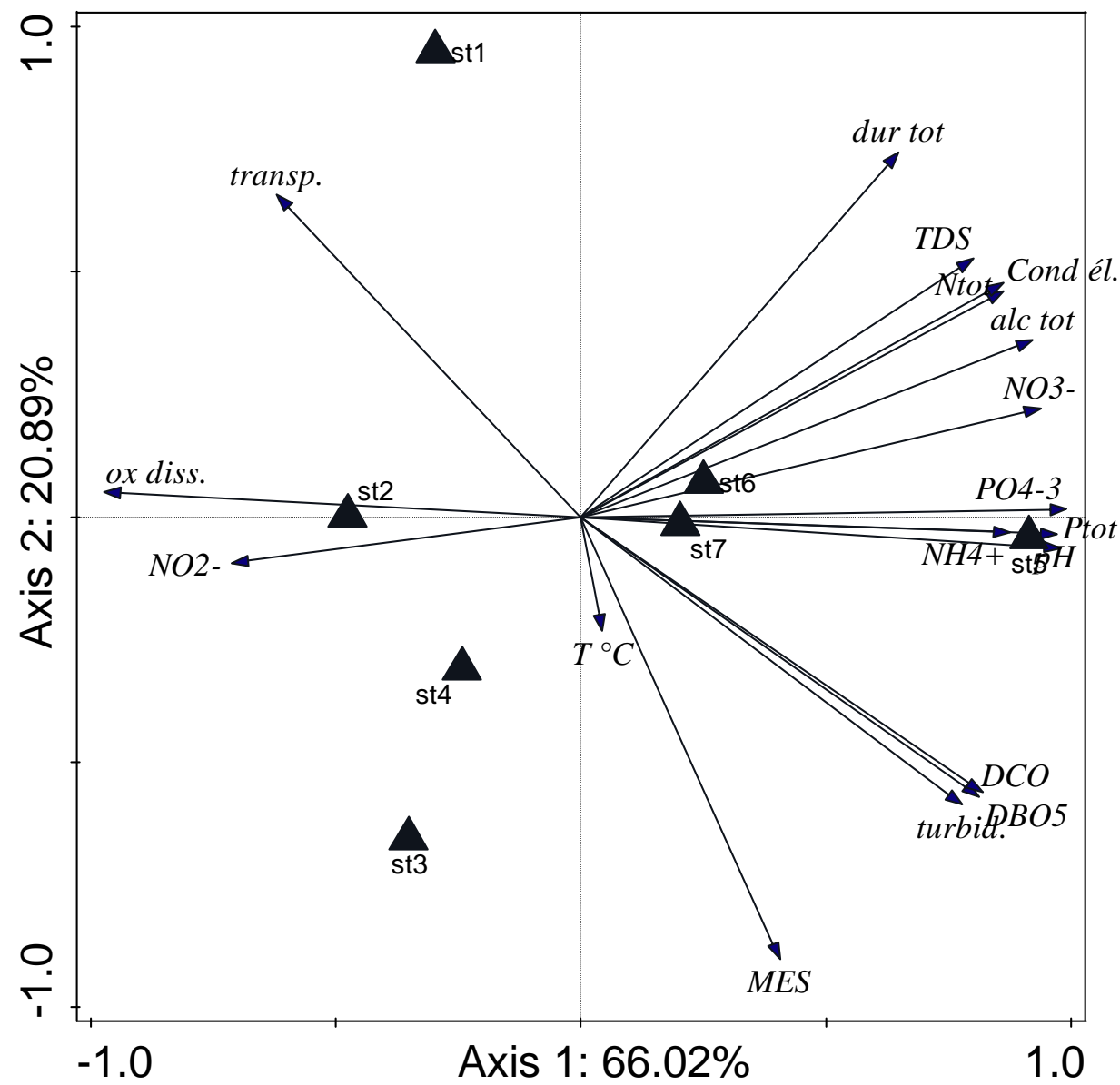

transp=transparence, ox diss=oxygène dissous, $\mathrm{NO}_{2}{ }^{-}=$nitrites, $\mathrm{NO}_{3}{ }^{-}=$nitrates, $\mathrm{DCO}=$ demande chimique en oxygène, $\mathrm{DBO}_{5}=$ demande biochimique en oxygène, MES=matières en suspension, turbid=turbidité, TDS=solides totaux dissous, cond él=conductivité électrique, alc tot=alcalinité totale, Ptot=phosphore total, Ntot=azote total.

Figure 2 : Analyse en Composantes Principales (ACP) des paramètres physico-chimiques.

Tableau 4: Classe de qualité des eaux de la rivière Kinyankonge.

\begin{tabular}{lcc}
\hline Classes de qualité & IPO & Valeurs obtenues de l'IPO \\
\hline Pollution organique nulle & $5,0-4,6$ & - \\
Pollution organique faible & $4,5-4,0$ & - \\
Pollution organique modérée & $3,9-3,0$ & $2,0-2,38$ \\
Pollution organique forte & $2,9-2,0$ & $1,91-1,95$ \\
Pollution organique très forte & $1,9-1,0$ &
\end{tabular}


Tableau 5 : Variation de l'indice de pollution organique (IPO) de juillet 2015 à juin 2016.

\begin{tabular}{|c|c|c|c|c|c|c|c|c|c|c|c|c|}
\hline Stations & Juil. & $\mathbf{A}$ & $\mathbf{S}$ & $\mathbf{O}$ & $\mathbf{N}$ & D & $\mathbf{J a}$ & $\mathbf{F}$ & Ma & $\mathbf{A}$ & Mai & Juin \\
\hline S 1 & 2,5 & 2,5 & 2,5 & 2 & 2 & 2,25 & 2,5 & 2,5 & 3 & 2,5 & 2 & 2,8 \\
\hline S 2 & 2 & & & 2 & 2,25 & 2,5 & 2,3 & 2 & 2,25 & 2 & 2 & 2,5 \\
\hline S 3 & 2,5 & 2,3 & 2,25 & 2,25 & 1,75 & 2,25 & 2 & 2 & 2,5 & 2,3 & 2 & 3,3 \\
\hline S 4 & 2 & 2 & 2 & 1,75 & 2 & 2,25 & 2 & 2 & 2 & 2 & 2 & 2,8 \\
\hline S 5 & 1,75 & 1,8 & 1,75 & 1,75 & 2 & 2,25 & 2 & 2,3 & 2,75 & 2 & 2 & 2,5 \\
\hline S 6 & 2 & 2 & 2 & 1,75 & 2 & 1,75 & 2,3 & 2 & 2,25 & 2,3 & 2 & 2 \\
\hline S 7 & 2 & 2 & 1,75 & 1,75 & 1,75 & 1,75 & 2 & 1,8 & 1,75 & 2 & 2 & 2,5 \\
\hline Moyen. & 2,11 & 2,10 & 2,04 & 1,89 & 1,96 & 2,14 & 2,16 & 2,09 & 2,36 & 2,16 & 2,00 & 2,63 \\
\hline E-T & 0,28 & 0,25 & 0,29 & 0,20 & 0,17 & 0,28 & 0,21 & 0,23 & 0,43 & 0,21 & 0,00 & 0,40 \\
\hline
\end{tabular}

Tableau 5 : Variation de l'indice de pollution organique (IPO) de janvier 2017 à juin 2017 (suite et fin).

\begin{tabular}{lcccccccc}
\hline Stations & Ja & F & Ma & A & Mai & Juin & Moyen. & E-T \\
\hline S 1 & 2,25 & 2,5 & 2,5 & 2 & 2,25 & 2,25 & 2,38 & 0,28 \\
S 2 & 2,25 & 2,25 & 2,5 & 2 & 2,25 & 2,5 & 2,22 & 0,2 \\
S 3 & 2 & 2,25 & 2,75 & 2,5 & 2 & 2,25 & 2,29 & 0,35 \\
S 4 & 2,25 & 2,25 & 2,5 & 2 & 2 & 2 & 2,1 & 0,24 \\
S 5 & 1,66 & 1,66 & 1,66 & 1,66 & 2 & 1,66 & 1,95 & 0,32 \\
S 6 & 1,66 & 1,66 & 2 & 1,66 & 1,66 & 1,66 & 1,93 & 0,22 \\
S 7 & 1,75 & 2 & 2 & 2 & 2 & 1,66 & 1,91 & 0,2 \\
Moyen. & 1,97 & 2,08 & 2,27 & 1,97 & 2,02 & 2 & & \\
E-T & 0,28 & 0,32 & 0,39 & 0,28 & 0,2 & 0,35 & & \\
\hline
\end{tabular}




\section{DISCUSSION}

Les paramètres physico-chimiques ayant fait objet de la présente étude ont servi pour l'évaluation de la qualité des eaux de la rivière Kinyankonge ainsi que leur variation spatio-temporelle. La température de l'eau de surface est utilisée pour évaluer sa qualité. Elle influence les processus biologiques dans les systèmes aquatiques (Kadlec et Reddy 2001). La température est un facteur clé qui régule la croissance des populations de zooplancton (Hong et al., 2003). Les valeurs de la température relevées lors de cette étude sont proches des valeurs relevées pour les eaux des régions tropicales chaudes (Villanueva, 2004; Aka Marcel, 2005 ; Konan et al., 2008). Elles ne varient pas d'une station à l'autre mais varient en fonction des saisons. Ceci s'explique par la faible distance qui sépare les différentes stations qui ont été échantillonnées.

Le $\mathrm{pH}$, quant à lui, est influencé par l'environnement traversé par la rivière notamment, la composition minérale, le type de sol et la roche même (Korfali et Jurdi, 2011; Mmualefhe et Torto, 2011). Pour la présente étude, une variation spatiotemporelle est observée de l'amont vers l'aval. À partir de la station S5, le pH est élevé suite au rejet d'effluents de nature alcaline provenant de la STEP et qui sont déversés dans la rivière Kinyankonge. Une augmentation du pH est aussi notée aux mois de juillet, août et septembre correspondant à la saison sèche. Une légère diminution est observée en saison pluvieuse suite aux pluies qui diluent les eaux. Le $\mathrm{pH}$ de la rivière Kinyankonge a varié entre 7,35 (S2) et 9,32 (S5). Ces valeurs se rapprochent de celles rapportées pour les eaux superficielles, soit entre 6,5 et 9,5 (Masamba et Mazvimavi, 2008 ; Nanituma, 2011). Les valeurs moyennes de $\mathrm{pH}$ mesurées aux stations $\mathrm{S} 6$ et S7 étaient respectivement de 7,89 et 7,97. Une légère augmentation est observée suite aux produits basiques déversés dans la rivière en provenance de la savonnerie. Elles avoisinent celles que Flura et al. (2016) ont relevé sur la rivière Meghna ghat (Bangladesh). Ces valeurs concordent aussi avec celles relevées sur d'autres cours d'eau qui traversent les zones à activités anthropiques intenses (Slim et al., 2000; Khalaf, 2003). Selon Blinda (2007), des $\mathrm{pH}$ compris entre 5 et 9 permettent un développement normal de la faune et de la flore.

Pour l'oxygène dissous qui est un paramètre important dans l'évaluation de la qualité des eaux, une variation spatiotemporelle est observée. Les stations amont présentent des valeurs moyennes relativement élevées; ce qui implique que l'eau est bien oxygénée. Une telle eau est propice au développement de la faune aquatique en général et du zooplancton en particulier. A la station S5, la présence de matières organiques oxydables a entraîné une diminution de la concentration en oxygène dissous en raison de l'épuisement de l'oxygène par décomposition aérobie des déchets organiques par des microorganismes. Une observation similaire a été faite par Dallas et Day (2004) dans les écosystèmes fluviaux d'Afrique du Sud. Tepe et Mutlu (2005) rattachent l'augmentation de l'oxygène dissous aux ruissellements élevés observés pendant la saison pluvieuse. La valeur moyenne d'oxygène dissous

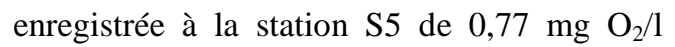
classe l'eau dans la catégorie des eaux médiocres selon Bliefert et Perraud (2001). Une telle eau ne permet pas une adaptation des espèces zooplanctoniques très diversifiées, seules résistent les espèces qui supportent une pollution organique élevée.

Les stations localisées en amont de la rivière Kinyankonge présentent des valeurs élevées de transparence et d'oxygène dissous par opposition aux stations situées en aval dont les valeurs sont plus basses. Neves et al. (2003) ont rapporté que l'augmentation de la transparence de l'eau conduit à l'augmentation de la diversité zooplanctonique. En effet, la clarté de l'eau a une incidence sur la quantité d'oxygène produit par le milieu. Une eau claire permet à la lumière de pénétrer davantage et favoriser ainsi la photosynthèse. On peut donc admettre que le cours d'eau en amont de la rivière Kinyankonge est en bon état. Ces observations sont semblables à celles de Chouti et al. 
(2010). Ces auteurs indiquent que la teneur en oxygène donne des indications sur la santé des cours d'eau et permet, entre autres, d'évaluer la quantité d'oxygène nécessaire pour les organismes aquatiques.

Par ailleurs, la conductivité électrique mesurée dans cette étude respectivement aux stations S1, S5, S6 et S7 pendant la saison sèche (juillet, août et septembre 2015, juin 2016 et janvier 2017) est élevée. Il est observé qu'en cette période, les valeurs élevées sont dues à la concentration élevée des ions dissous dans l'eau. Le pic observé à la station $\mathrm{S} 5$ indique une forte ionisation à ce niveau. $\mathrm{Ce}$ phénomène a été rapporté par certains auteurs tels que Kambole (2003); Atibu et al. (2013); Mubedi et al. (2013) et Tshibanda et al. (2014) qui affirment que la conductivité électrique élevée indique également le degré de minéralisation de l'eau. Cette minéralisation est fonction de la solubilité des composés dissous et dissociés; ce qui prédit une teneur élevée en ions. Ben Moussa et al. (2012) affirment qu'une faible conductivité électrique pour un cours d'eau est également synonyme d'une faible minéralisation des sels en présence dans le milieu. Ainsi, en pleine saison pluvieuse, elle diminue suite à la dilution des sels et des ions due aux pluies. Cette observation concorde avec les observations de Paizis (2012) dans le Delta d'Okavango au Nord-Ouest de Botswana. Néanmoins, les valeurs relativement élevées de conductivité électrique dans certaines stations d'échantillonnage, sont dues au fait que la rivière servait de conduite des effluents urbains, de fertilisants, des eaux usées ou autres déchets provenant des ménages. Lung'aiya et al. (2000) et Gikuma-Njuru et Hecky (2005) ont fait les mêmes observations sur le lac Victoria.

En ce qui concerne les TDS, ils sont équivalents à la minéralisation totale qui correspond à la somme des anions et des cations présents dans les eaux (Kambiré et al., 2014). Les solides totaux dissous (TDS) et la conductivité électrique sont fortement corrélés pour la présente étude. Cette observation concorde avec celle faite par Ewa et al. (2011) qui ont enregistré une conductivité électrique en forte corrélation avec les TDS dans la rivière d'Omoku Creek, au Nigeria. Certains auteurs affirment que la conductivité électrique élevée ne favorise pas le développement et la prolifération du zooplancton (Monney et al., 2016).

Les nitrates proviennent de l'oxydation des ammoniums en nitrites puis en nitrates. L'ion nitreux est instable. Il se transforme en nitrates qui sont l'état final de l'oxydation de l'ammonium. La présence des nitrates dans les cours d'eau est due, soit au lessivage des sols agricoles, soit aux réactions oxydatives de l'azote ammoniacal et des nitrites (Khalaf, 2003; Saad et al., 2004 ; Khalaf et al., 2007). Certaines études ont montré que lorsque dans une rivière la concentration en ions ammoniums est comprise entre $0,1-3 \mathrm{mg} / \mathrm{l}$ $\mathrm{NH}_{4}{ }^{+}$, l'eau est polluée (Belhaouari et al., 2017). Pour la présente, les ammoniums ont varié de $0,67-1,88 \mathrm{mg} / 1 \mathrm{NH}_{4}{ }^{+}$.

Les valeurs élevées de phosphore total à la station S5 trouve leur origine à la station d'épuration de Buterere. La moyenne a varié de 1,27 à $3,33 \mathrm{mg} / \mathrm{l} \mathrm{P}$ tot. Or, les eaux dont les valeurs sont supérieures à $3 \mathrm{mg} / \mathrm{l} \mathrm{P}$ tot sont de mauvaise qualité. Il en est de même pour l'azote total avec des valeurs élevées relevées à cette station. Cette station déverse des produits azotés dans la rivière Kinyankonge.

Quant aux phosphates, ils proviennent de la décomposition de la matière organique ou du lessivage des terres agricoles (Slim et al., 2000; Khalaf, 2003; Saad et al., 2004 ; Khalaf et al., 2007). À la station S5, les valeurs moyennes relevées étaient plus élevées comparées aux autres stations, ce qui montre que la station d'épuration contribue de façon importante à l'augmentation des concentrations des paramètres chimiques en déversant dans la rivière les effluents non traités. Ce constat réconforte les observations de Niyungeko (2011) qui a rapporté des valeurs élevées des paramètres de pollution au bassin de maturation de la station d'épuration de Buterere et qui sont déversés directement dans la rivière Kinyankonge. Quant aux autres stations, les phosphates proviennent d'eaux usées domestiques. En effet, beaucoup de 
produits domestiques d'entretien tels que les savons et les détergents contiennent des éléments phosphorés. Ils peuvent également avoir pour origine le lessivage des sols à travers l'érosion des terres agricoles en provenance des bassins versants de la ville de Bujumbura comme d'autres études l'ont révélé (Majaliwa et al., 2004 ; Azanga et al., 2016). Selon Foto Menbohan et al. (2012), un déversement d'effluents de nature organique entraîne une augmentation de la conductivité, de l'azote ammoniacal, des orthophosphates, des matières en suspension et de la turbidité défavorable au développement du zooplancton. Les eaux provenant de la station S5 sont de mauvaise qualité et ne favorise pas la diversité zooplanctonique. L'alcalinité totale est élevée à la station S5 et pendant la saison sèche. Elle est liée au système tampon développé par les carbonates et les bicarbonates qui libèrent les carbonates susceptibles de rehausser le $\mathrm{pH}$ de l'eau (Wetzel, 2001). Une alcalinité supérieure à 40 $\mathrm{mg} / \mathrm{l}$ est un bon indicateur d'une grande productivité du milieu, ce qui s'accorde avec les résultats de la présente étude. Elle a varié de $137,3 \mathrm{mg} / \mathrm{l}$ à $527,6 \mathrm{mg} / \mathrm{l}$ enregistrées respectivement à la station $\mathrm{S} 3$ et à la station S5.

La dureté totale enregistrée dans les différentes stations échantillonnées est élevée, les stations S1 et S5 ont enregistré les valeurs élevées, ce qui est dû à une concentration élevée en ions Calcium et Magnésium. La dureté totale, la conductivité électrique et les TDS sont liés.

La troisième station a enregistré une moyenne élevée de MES et cette valeur élevée est due aux exploitants du sable qui ont perturbé la qualité des eaux de la rivière Kinyankonge ainsi qu'aux eaux de ruissellement chargées de débris et matières qui ne décantent pas. Le pic a été observé au mois de mars 2016 correspondant à la période des pluies. Les MES sont fortement liées à la turbidité. Selon Pitt et al. (2005) et Wong (2006), plusieurs polluants peuvent être associés aux eaux de ruissellement comme les métaux toxiques et les nutriments se liant avec les particules de sédiments fins avec une incidence négative sur le milieu récepteur, ce qui présente un énorme danger pour les organismes aquatiques.

$\mathrm{La} \mathrm{DCO}$ et la $\mathrm{DBO}_{5}$ sont fortement liées puisque le rapport $\mathrm{DCO} / \mathrm{DBO}_{5}$ est un indicateur de la biodégradabilité de l'effluent. Si ce rapport est inférieur à 2, l'effluent est facilement biodégradable. Les valeurs moyennes sont plus élevées à la station S5 et évoluent de la station S1 à la station S5 pour diminuer à la station S6 avant d'augmenter légèrement à la station $\mathrm{S} 7$. Ces valeurs élevées sont, pour la plupart, dues au lessivage de la matière organique accumulée en saison sèche vers la rivière. La variation spatiale de la DCO était identique à celle de la $\mathrm{DBO}_{5}$, D'autres études ont trouvé une corrélation positive entre ces deux paramètres (Yapo et al., 2009). Ces deux paramètres sont fortement liés. Les concentrations moyennes de la DCO et de la $\mathrm{DBO}_{5}$ relevées lors de cette étude sont supérieures aux normes de rejet dans une rivière fixées à $80 \mathrm{mg} \mathrm{O}_{2} / \mathrm{l}$ et $25 \mathrm{mg} \mathrm{O} \mathrm{O}_{2} / \mathrm{l}$ respectivement (Bliefert et Perraud, 2001). Pour le cas présent, l'eau est située dans la catégorie «hors classe » ou très mauvaise selon Bliefert et Perraud (2001). Elle ne permet pas le développement des organismes aquatiques notamment les invertébrés.

Cette étude a montré que la pollution de la rivière Kinyankonge a augmenté d'amont en aval puisque, sur son parcours, elle charrie des polluants d'origines diverses vers le milieu récepteur final qui est le lac Tanganyika. Une telle situation a été rapportée par Ntakiyiruta et al. (2017) sur la rivière Ntahangwa au Burundi.

Ce constat est confirmé par l'indice de pollution organique (IPO) utilisé dans la présente étude pour évaluer la charge organique de la rivière. En effet, les faibles valeurs de l'IPO obtenues aux stations situées en aval de la rivière Kinyankonge confirment également les teneurs élevées des paramètres physico-chimiques relevées à ce niveau, tandis que les fortes valeurs de l'IPO obtenues aux stations situées en amont de la rivière Kinyankonge confirment les teneurs des paramètres physico-chimiques relevées qui y sont faibles. L'IPO a permis de dégager que la 
pollution des eaux de la rivière Kinyankonge est une pollution organique.

\section{Conclusion}

Certains paramètres physico-chimiques des eaux de la rivière Kinyankonge ont présenté des valeurs qui dépassent les normes de rejet dans le milieu récepteur final qui est le lac Tanganyika. L'indice de pollution organique a montré que les stations amont sont de pollution organique forte et les stations situées en aval sont de très forte pollution organique. Cette pollution provient des rejets ménagers des habitations riveraines de la rivière Kinyankonge et des effluents en provenance de la STEP et de la Savonnerie. La pollution de la STEP vient des ménages raccordés au réseau d'égouttage. Les produits de lavage, une fois versés dans les conduites, sont acheminés vers la STEP. C'est pourquoi la plupart des paramètres mesurés à ce niveau sont élevés, ce qui présente un danger pour les espèces de la flore et de la faune qui vivent dans cet écosystème aquatique. Cette pollution ne favorise pas le développement et l'abondance des invertébrés comme le zooplancton. Une étude approfondie à long terme permettra de mettre en exergue l'impact de cette pollution sur la biodiversité de cet écosystème aquatique.

\section{CONFLIT D'INTERETS}

Les auteurs de cet article déclarent qu'ils n'y a aucun conflit d'intérêts pour cette publication.

\section{CONTRIBUTIONS DES AUTEURS}

$\mathrm{SB}$ et EM ont conçu le protocole de l'étude; SB, EM et GN ont collecté les données et analysé au laboratoire; SB, EM, $\mathrm{CS}, \mathrm{EB}$ et GN ont analysé les données; SB, $\mathrm{EM}, \mathrm{CS}, \mathrm{EB}, \mathrm{GN}$ et $\mathrm{CAB}$ ont participé à la rédaction de l'article.

\section{REMERCIEMENTS}

Les auteurs remercient et les membres du Laboratoire de Recherche en Aquaculture et en Biologie et Ecologie Aquatiques de l'Ecole d'Aquaculture de la Vallée de l'Université Nationale d'Agriculture (Bénin).

\section{RÉFÉRENCES}

APHA (American Public Health Association). 1995. Standard Methods for the Examination of Water and Waste Water $\left(19^{\text {th }}\right.$ edn). APHA: Washington D.C., USA; 874.

Aquerec Project. 2006. Work package 2 final report, Delivrable D15, EVK1-CT20026-00130. Integrated concepts for reuse of upgraded wastewater. URL WWW.aquerec.org.

Atibu EK, Devarajan N, Thevenon F, Mwanamoki PM, Tshibanda JB, Mpiana PT, Prabakar K, Mubedi JI, Walter W, Poté J. 2013. Concentration of metals in surface water and sediment of Luilu and Musonoie Rivers, Kolwezi-Katanga, Democratic Republic of Congo. Appl. Geochem., 39: 26-32.

Azanga E, Majaliwa M, Kansiime F, Mushagalusa N, Korume K, Tenywa MM. 2016. Land-use and land-cover, sediment and nutrient hotspot areas changes in Lake Tanganyika basin. African Journal of Rural Development, 1(1): 75-90.

Belhaouari B, Belguermi A, Achour T. 2017. Protection des eaux de surfaces continentales en Algérie : quelle stratégie faut-il adopter pour les dix prochaines années? Larhyss Journal, 31(7-17).

Ben Moussa A, Chahlaoui A, Rour El H. 2012. Évaluation de la pollution physicochimique des eaux de l'Oued Khoumane (Moulay Idriss Zerhoun, Maroc). Int. J. Biol. Chem. Sci., 6(6): 7096-7111. DOI : http://dx.doi.org/10.4314/ijbcs.v6i6.44.

Bliefert C, Perraud R. 2001. Chimie de l'Environnement: Eau, Air, Sols, Déchets. Paris, Deboeck, Université, 477p.Blinda M. 2007. Pollution tellurique du littoral Nord-Ouest du Maroc entre Tanger et Tétouan: Caractérisation, impact sur l'environnement et proposition de solutions. Thèse de doctorat, université Mohammed V, Faculté des Sciences, Rabat, 194 pp.

Buhungu S, Houssou AM, Montchowui E, Ntakimazi G, Vasel JL, Ndikumana T. 
2017. Etablissement du pollutogramme et de l'hydrogramme de la rivière Kinyankonge, Burundi. Int. J. Biol. Chem. Sci., 11(3): 1386-1399. DOI : https://dx.doi.org/10.4314/ijbcs.v11i3.37

Chouti W, Mama D, Alapini F. 2010. Études des variations spatio-temporelles de la pollution des eaux de la lagune de PortoNovo (Sud-Bénin). Int. J. Biol. Chem. Sci., 4(4): 1017-1029. DOI: http://dx.doi.org/10.4314/ijbcs.v4i4.630.

Dallas HF, Day J. 2004. The effect of water quality variables on aquatic ecosystems: a review. WRC report no.: TT224/04, $221 \mathrm{pp}$.

Dudgeon D, Angela HA, Gessner MO, Kawabata ZI, Knowler DJ, Lévêque C, Naiman RJ, Prieur RAH, Soto D, Stiassny MLJ, Sullivan CA. 2006. Freshwater biodiversity: importance, threats, status and conservation challenges. Biol. Rev., 81: 163-182. DOI: $10.1017 /$ S1464793105006950.

El Morhit M. 2009. Hydrochimie, éléments traces métalliques et indice écotoxicologiques sur les différentes composantes d'un écosystème estuarien (Bas Lukkos), Thèse de doctorat de l'Université de Mohammed V-Agdal de Rabat, Maroc, 260p.

Ewa EE, Iwara AI, Adeyemi JA, Eja EI, Ajake AO, Otu CA. 2011. Impact of Industrial Activities on Water Quality of Omoku Creek. Sacha J Environ Studies, 1(2):8-16.

Flura, Mohammad AA, Akhery N, Mohosena BT, Masud HK. 2016. Physico-chemical and biological properties of water from the river Meghna, Bangladesh. International Journal of Fisheries and Aquatic Studies, 4(2): 161-165.

Foto Menbohan S, 2012. Recherches écologiques sur le réseau hydrographique du Mfoundi (Yaoundé): Essai de biotypologie. Thèse de Doctorat d'État en Biologie des Organismes Animaux,Université de Yaoundé I. Cameroun, $175 \mathrm{p}$ +annexes.

Hong WL, Syuhei B, Tsutomu I, Takashi M. 2003. Effect of temperature on development, growth and reproduction in the marine copepod Pseudocalanus newmani at satiating food condition. Journal of Plankton Research, 25(3): 261-271.

Kadlec RH, Reddy KR. 2001. Temperature effects in treatment wetlands. Water Environment Research, 73: 543-557.

Kambiré O, Adingra AA, Eblin SG, Aka N, Kakou AC, Koffi-Nevry R. 2014. Caractérisation des eaux d'une lagune estuarienne de la côte d'Ivoire: la lagune Aby. Larhyss Journal, 20: 95-110.

Kambole MS. 2003. Managing the water quality of the Kafue River. Phys. Chem. Earth., 28(20-27): 1105-1109. DOI: 10.1016/j.pce.2003.08.031.

Khalaf G. 2003. Etude biocénotique et qualité des eaux de quatre cours d'eau côtiers libanais Nahr Ibrahim, Nahr El Kalb, Nahr Antélias et Nahr Beyrouth : biologie et écologie de Capoeta damascina Günther 1868 (Poisson, Cyprinidae). Thèse de doctorat, Université Perpignan, 20 p. + annexes.

Khalaf G, Slim K, Saad Z, Nakhlé K. 2007. Evaluation de la qualité biologique des eaux du Nahr el Jaouz (Liban) : application des méthodes indicielles. Bull. Mens. Soc. Linn. Lyon., 76(9-10): 255- 268.

Konan KS, Aka MK, Adingra AA, Dongui BK, Gnakri D. 2008. Variations saisonnières des paramètres abiotiques des eaux d'une lagune tropicale : la lagune de Grand-Lahou, Côte d'Ivoire, European Journal of Scientific Research, 21(3): 376-393.

Korfali SI, Jurdi M. 2011. Suitability of surface water for domestic water use. Awali River Case Study: European Water, 35: 3-12.

Kouassi, Aka M. 2005. Hydrochimie et qualité des eaux de deux lagunes tropicales de Côte d'Ivoire (Ebrié, Grand Lahou). Thèse de Doctorat, Université de Cocody, Abidjan, 147 p.

Leclercq L. 2001. Les eaux courantes : caractéristiques et moyens d'étude, dans les zones humides. Actes des colloques 
organisés en 1996 par le Ministère de la Région Wallonne dans le cadre de l'Année Mondiale des Zones Humides, Jambes, Région Wallonne, DGRNE. pp. 67-82.

Lung'aiya HBO, M'Harzi A, Tackx M, Gichuki J, Symoens JJ. 2000. Phytoplankton Community Structure and Environment in the Kenyan Waters of Lake Victoria, Freshwater Biology, 43: pp 529-543.

Majaliwa JGM, Magunda MK, Tenywa MM. 2004. Non point pollution loading in a selected micro catchment of the Lake Victoria basin. In Proceedings of the Ninth International Symposium on river sedimentation, Yinchang, China, 2206$2211 \mathrm{p}$

Masamba WRL, Mazvimavi D. 2008. Impact on water quality of land uses along

Thamalakane-Boteti River: an outlet of the Okavango Delta. Physics and Chemistry of the Earth, 33: 687-694.

Mc Kinney ML. 2002. Urbanization, biodiversity and conservation. Biosci. 52, 883-890.Mohammad MJ, Mazareh N. 2003. Changes in soil fertility parameters in response to irrigation of forage crops with secondary treated wastewater. Communication in Soil Science and Plant Analysis, 34(9-10): 1281-1294.

Monney Attoubé Ida, Issa Nahoua Ouattara, Raphaël N'doua Etilé, Maryse N'guessan Aka, Mamadou Bamba, Tidiani Koné. 2016. Distribution du zooplancton en relation avec les caractéristiques environnementales de quatre rivières côtières du Sud-est de la Côte d'Ivoire (Afrique de l'ouest). Journal of Applied Biosciences, 98: 9344-9353.DOI: http://dx.doi.org/ 10.4314/ jab.v98i.1.10.

Mmualefhe LC, Torto N. 2011. Water quality in the Okavango Delta. Water SA 37: 411-418.Mubedi JI, Devarajan N, Faucheur SL, Kayembe JM, Atibu EK, Periyasamy S, Kandasamy P, Mpiana PT, Wildi W, Poté J. 2013. Effects of untreated hospital effluents on the accumulation of toxic metals in sediments of receiving system under tropical conditions: case of South India and Democratic Republic of Congo. Chemosphere, 93: 1070-1076. DOI: 10.1016/j.chemosphere.2013.05.08 0

Nanituma M. 2011. Contribution à l'effort de production et de distribution de l'eau potable en R.D.C. " cas de la ville de Kinshasa". Thèse de doctorat en Sciences chimiques, Université de Kinshasa (R.D.C).

Neves IF, Rocha D, Roche KF, Pinto AA. 2003. Zooplankton community structure of two marginal lake of river (Cuiaba) (Mato, Grosso, Brazil) with analysis of rotifer and Cladocera diversity. Braz. J. Biol., 63(2): 329-343.

Niyongabo H. 2006. Intégration des enjeux social et quantitatif pour la maîtrise de l'assainissement liquide en Afrique Tropicale : Application à la ville de Bujumbura. Ph.D. Thesis, Faculté des Sciences Appliquées, Université de Liège, $293 \mathrm{p}$.

Niyungeko C. 2011. Evaluation de l'efficacité épuratoire de la station de lagunage de

Buterere. Mémoire présenté en vue de l'obtention d'un Diplôme de Master Complémentaire en Sciences de l'Environnement, Facultés des Sciences, Université du Burundi, 71p.

Njuru P, Hecky RE. 2005. Nutrient concentrations in Nyanza Gulf, Lake Victoria, Kenya: light limits algal.Nsavyimana G, 2014. Modélisation des processus physiques et biologiques dans des fosses septiques et voies de valorisation des boues de vidange: Application à Bujumbura-Burundi. Thèse de doctorat. Université de Liège, $427 p$.

Ntakiyiruta P, Nahimana D, Ntakimazi G. 2017. Contribution à l'estimation quantitative des nutriments apportés par la rivière Ntahangwa au lac Tanganyika. Afrique Science, 13(1): 360 - 370.

Paizis N. 2012. Investigation of diel variations of water quality and stable carbon isotopes 
across the Okavango Delta, northwest Botswana. Poster presented at 2012 GSA Annual Meeting and Exposition, 4-7 November, Charlotte, North Carolina. In: Geological Society of America Abstracts with Programs, 44(7): 394.

Perroux AS. 2006. Intérêt des sédiments détritiques endokarstiques en tant qu'archive naturelle ? Discussion autour des dépôts lacustres souterrains (Grottes de Choranche-Vercors). Kartologia, 47: 7 20.

Pitt R, Williamson D, Voorhees. 2005. Review of historical street dust and dirt accumulation and wash off data. In Dans Effective Modeling of Urban Systems, Monograph 13, James, Irvine, McBean, Pitt (eds), CHI: Guelph, Ontario.

Rodier J. 2005. Analyse de l'eau: eaux naturelles, eaux résiduaires, eaux de mer : Chimie, Physicochimie, Biologie, interprétation des résultats, Paris : Dunod.

Saad Z, Slim K, Khalaf G, Elsamad O. 2004. Impact des rejets des eaux résiduaires sur la qualité physico-chimique et algologique du Nahr Antélias. Bulletin de la Société Neuchâteloise des Sciences Naturelles, 127: 69-82.

Sala F, Osvaldo E, Stuart Chapin III, Juan J, Armesto, Berlow E, BloomPeld J, Dirzo R, Huber-Sanwald E, Laura F, Huenneke, Robert B, Jackson, AKinzig, Rik L, David M, Lodge, Harold A, Mooney, Martõ ON, LeRoy P, Martin T, Sykes, Brian H, Walker, Marilyn W, Diana H, Wall. 2000. Global Biodiversity Scenarios for the Year 2100. Science, 287: 1770; DOI: 10.1126/Science.287.5459.1770.

Simpi B, Hiremath SM, Murthy KNS. 2011. Analysis of Water Quality Using PhysicoChemical Parameters Hosahalli Tank in Shimoga District, Karnataka, India. Global Journal of Science Frontier Research, 11(3): 31-34.

Slim K, Saad Z, Khalaf G. 2000. Estimation de la qualité des eaux du Nahr Beyrouth. Utilisation de l'indice diatomique de polluo-sensible (IPS). Cah. Ass. Sci. Eur. Eau et Santé, 5: 51-60.

Tepe Y, Mutlu E. 2005. Physico-chemical characteristics of Hatay Harbiye Spring water, Turkey. J. Inst. Sci. Tech. Dumlupinar University, 6: 77-88.

TS Cox, M Bender, C Picone, DL Van Tassel, JB Holland, EC Brummer, BE Zoeller, AH Paterson, W Jackson. 2002. Breeding Perennial Grain Crops, Critical Reviews in Plant Sciences, 21 (2): 59-91.

Tshibanda JB, Devarajan N, Niane B, Mwanamoki PM, Atibu EK, Mpiana PT, Prabakar K, Mubedi JI, Kabele CG, Wildi W, Poté J. 2014. Microbiological and physicochemical characterization of water and sediment of an urban river: N'Djili River, Kinshasa, Democratic Republic of the Congo. Sustainability of Water Quality and Ecology. DOI: 10.1016/j.swaqe.2014.07.001.

Villanueva MC S. 2004. Biodiversité et relations trophiques dans quelques milieux estuariens et lagunaires de l'Afrique de l'Ouest : adaptations aux pressions environnementales, Thèse de Doctorat de l'Institut National Polytechnique de Toulouse, Toulouse, $246 \mathrm{p}$.

Vindimian E. 2006. Problématique des substances à risque. In: Conférence Eau et Santé. Eaux pluviales et assainissement : nouvelles préoccupations sanitaires, GRAIE, Grand-Lyon et ASTEE. 10.10.2006, Lyon-Villeurbanne, 2006 pp. $55-73$.

Welch sp. 1948. Limnological Methods. McGraw Hill Book Co.: New York, USA, 381.

Wetzel RG. 2001. Limnology: Lake and River Ecosystems (3rd edn). Academic Press: New York; 1006.

Wong THF. 2006. Australia Runoff Quality-A Guide to Water Sensitive Urban Design. Engineers Australia: Melboume.

Yapo BO, Mambo V, Seka A, Yapi AD, Houenou P. 2009. Caractérisation par fractionnement gravimétrique de la matière organique contenue dans les eaux usées : application à l'étude de la biodégradabilité, Journal de la Société Ouest-Africaine de Chimie, 27: 21 - 37. 